\title{
O MODELO CONCEITUAL DE SISTEMAS ABERTOS INTERATUANTES E A TEORIA DE ALCANCE DE METAS DE IMOGENE KING ${ }^{1}$
}

Thereza Maria Magalhães Moreira²

Thelma Leite de Araújo $^{3}$

Moreira TMM, Araújo TL. O modelo conceitual de sistemas abertos interatuantes e a teoria de alcance de metas de Imogene King. Rev Latinoam Enfermagem 2002 janeiro-fevereiro; 10(1):97-103.

O trabalho descreve o Modelo Conceitual de Sistemas Abertos Interatuantes e a Teoria de Alcance de Metas de Imogene King, refletindo sobre o cuidar a partir dessa proposta. Foi realizado a partir de extenso estudo da estrutura teórica de King, assim como de sua aplicação junto aos pacientes. Foi possível observar que a estrutura teórica fornece um arcabouço teórico-conceitual necessário ao cuidado de enfermagem humanizado, estando atualizada e inserida no contexto atual de saúde.

DESCRITORES: teoria de enfermagem, enfermagem

\section{THE CONCEPTUAL MODEL OF INTERACTIVE OPEN SYSTEMS AND THE THEORY OF GOAL ATTAINMENT BY IMOGENE KING}

This work describes the Conceptual Model of Interactive Open Systems and the Theory of Goal Attainment by Imogene King by reflecting on the care process based on this proposal. It was accomplished as a result of the extensive study of King's theoretical structure and its application with patients. It was possible to observe that the theoretical structure supplies the necessary theoretical and conceptual framework which is necessary for humanized nursing care. Also, it remains updated and included in the current context of health care.

KEY WORDS: nursing, nursing theory

\section{MODELO CONCEPTUAL DE SISTEMAS ABIERTOS INTERACTUANTES Y LA TEORÍA DE ALCANCE DE METAS DE IMOGENE KING}

El trabajo describe el Modelo Conceptual de Sistemas Abiertos Interactuantes y la Teoría de Alcance de Metas de Imogene King, reflexionando sobre el cuidado a partir de ésta propuesta. Fue realizado a partir de un estudio exhaustivo de la estructura teórica de King, así como de su aplicación a los pacientes. Fue posible observar que la estructura teórica proporciona una base teórica-conceptual necesaria para el cuidado de enfermería humanizado, estando actualizada e incluida en el contexto actual de salud.

DESCRIPTORES: teoría de enfermería, enfermería

\footnotetext{
${ }^{1}$ Trabalho integrante da dissertação de Moreira (1999), desenvolvido no Projeto Cuidado em Saúde Cardiovascular, financiado pelo CNPq; ${ }^{2}$ Enfermeira, Mestre em Enfermagem, Doutoranda em Enfermagem pela Universidade Federal do Ceará; ${ }^{3}$ Enfermeira, Doutor em Enfermagem, Coordenador do Projeto, e-mail: therezamaria@yahoo.com.br
} 


\section{INTRODUÇÃO}

As teorias de enfermagem são uma construção, a partir de uma prática idealizada, que visa aperfeiçoar a assistência ${ }^{(1)}$. Um modelo conceitual é um arcabouço ou quadro de conceitos e proposições gerais e abstratas, que fornece uma estrutura de referência ou perspectiva distinta em um domínio de investigação especifico $^{(2)}$

Dada a necessidade de fundamentar as ações para 0 cuidado de enfermagem, o tema tem sido um dos assuntos mais discutidos atualmente ${ }^{(2-3)}$. Assim, o trabalho descreve o Modelo Conceitual de Sistemas Abertos Interatuantes e a Teoria de Alcance de Metas de Imogene King, refletindo sobre o cuidar a partir dessa proposta. Foi realizado a partir de extenso estudo da estrutura teórica de King, assim como de sua aplicação junto aos pacientes.

\section{DESCRIÇÃO DE UM MODELO CONCEITUAL}

O Modelo Conceitual utilizado ${ }^{(4)}$ é a estrutura conceitual sobre a qual repousa sua Teoria de Alcance de Metas. A autora é uma figura marcante na enfermagem mundial pela estruturação de seu modelo de Sistemas Abertos Interatuantes.

No Modelo Conceitual de Sistemas Abertos, a saúde é definida como ajuste contínuo a estressores no ambiente interno e externo pela otimização dos recursos da pessoa para alcançar um potencial máximo para viver. São determinados três sistemas interativos: o pessoal, interpessoal e social ${ }^{(4)}$.

- Sistema pessoal: é o tipo de sistema compreendido por um indivíduo em um ambiente. Esse sistema engloba os conceitos de percepção, ego, imagem corporal, crescimento, desenvolvimento, tempo e espaço.

- Sistema interpessoal: é formado pelo agrupamento de indivíduos em díades, tríades e pequenos e grandes grupos. Nesse sistema, são englobados os seguintes conceitos: papel, interação, comunicação, transação e estresse.

- Sistema social: dá-se pela reunião de grupos com interesses e necessidades especiais, formando organizações e compondo sociedades. Os conceitos relacionados neste sistema são: organização, autoridade, poder, status, tomada de decisão e papel.

A enfermagem é conceituada como percepção, pensamento, relacionamento e ação, frente ao comportamento dos indivíduos que vêm ao ambiente imediato e à realidade espacial e temporal, que compõe uma situação de enfermagem. Nessa, a enfermeira e o paciente estabelecem uma relação de enfrentamento aos estados de saúde e ajuste a atividades de mudança na vida diária, se a situação exigir. A enfermagem é, assim, um processo de ação, reação (resposta), interação e transação, pelo qual são dadas informações sobre as percepções da enfermeira e indivíduo na situação de enfermagem ${ }^{(4)}$.

Neste modelo conceitual, parte-se da suposição de que a meta da enfermagem é ajudar o indivíduo a manter sua saúde para que, dessa maneira, ele possa desempenhar bem seus papéis ${ }^{(4)}$, ou seja, é, sobretudo, o cuidado de seres humanos. A estrutura conceitual é identificada como sistemas abertos, intercomunicantes (Figura 1).

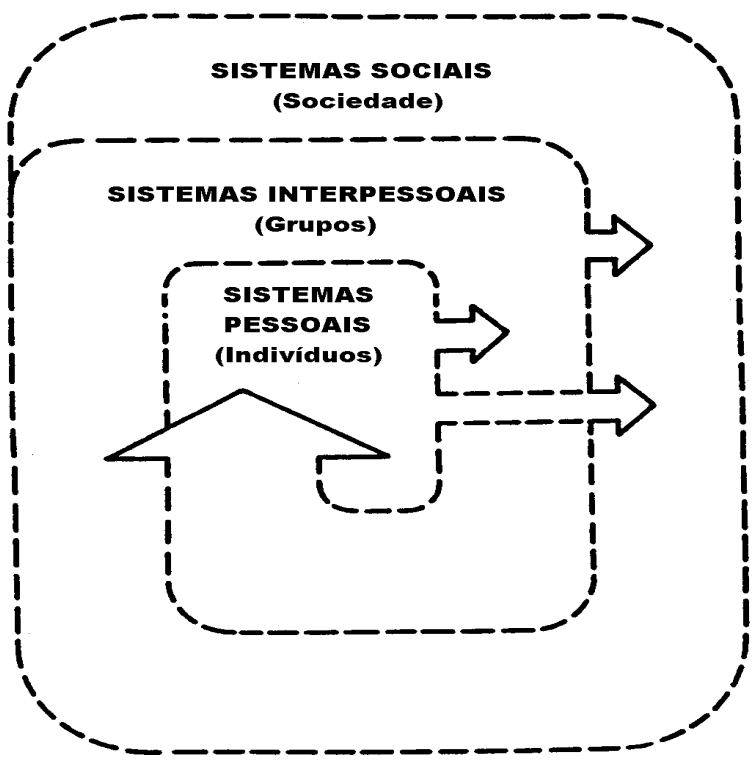

Figura 1 - Estrutura conceptual de Imogene M. King ${ }^{(4)}$

Supõe-se que as enfermeiras têm como funções ensinar, aconselhar e guiar indivíduos e grupos, ajudando-Ihes a manter sua saúde. Sua estrutura se baseia no fato de que o foco da enfermagem é o cuidado de seres humanos. Esses permanecem em contínua interação com o ambiente e com as pessoas que os cercam. Cada sistema se encontra em interação com os demais, garantindo 0 equilibrio individual e grupal. Pelo caráter de sistemas abertos, as alterações em um desses sistemas podem repercutir nos demais ${ }^{(4)}$.

Os conceitos relacionados nos sistemas pessoal, interpessoal e social fornecem uma rede conceitual sobre a qual repousa o modelo, traçando um emaranhado teórico que delimita cada sistema. No sistema pessoal, são definidos os conceitos ${ }^{(4)}$ :

Percepção, como a representação do real de cada ser humano. É universal porque todas as pessoas têm percepção, embora esta seja subjetiva, pessoal e seletiva. Sua ação está voltada ao presente e baseia-se na informação disponível. Os dados obtidos mediante os sentidos e a memória são organizados, interpretados e transformados. É um conceito muito importante às enfermeiras por permitir o desenvolvimento de uma base para juntar e interpretar informações. A percepção é base para o conceito de ego.

Self ou eu é um sistema aberto voltado a uma meta. É a concepção que o próprio indivíduo tem de quem e do que ele é. 
Sofre influências do passado e especula acerca do futuro. Um sistema pessoal é uma unificação que o self percebe, pensa, deseja, imagina, decide, identifica metas e seleciona as que pretende alcançar. 0 self é um ser reativo por natureza. Toda enfermeira e paciente tem um self, mas é a consciência desse que ajuda a tornar um indivíduo sensivel. Cada self é uma pessoa inteira, com crescimento e desenvolvimento na estrutura corporal e cognitiva humana.

Crescimento e desenvolvimento são conceituados como algo que inclui mudanças celulares, moleculares e comportamentais nos indivíduos. São os próprios processos de vida, nos quais as pessoas vão de um potencial, para a conquista de atualização do self. Conhecer o modo como as pessoas crescem e se desenvolvem, e seu eu, ajuda as enfermeiras a entenderem as pessoas com problemas na imagem corporal.

A imagem corporal é definida como o componente integrante do crescimento e desenvolvimento que é influenciado pelo self. É a maneira pela qual as pessoas percebem, não só o seu corpo, mas também as reações dos outros à sua aparência. É um conceito extremamente pessoal e subjetivo. É importante que as enfermeiras entendam o conceito de imagem corporal e suas alterações, para que possam ajudar àqueles que apresentam alterações de autoimagem.

O espaço é um componente essencial no modelo conceitual de sistemas abertos de King. É definido pela sua área física (território) e pelos comportamentos dos que o ocupam. Difere do sistema pessoal porque este não tem limites visíveis. 0 espaço é universal porque todas as pessoas têm algum conceito de espaço, mas é pessoal e situacional, na medida em que é percebido pelas pessoas e alterado de uma situação a outra. É dimensional (tem função de área, volume, distância e tempo) e transacional (determina as transações entre os seres humanos e o ambiente). É necessário que as enfermeiras tenham claro um conceito de espaço para aperfeiçoar o entendimento de si mesma enquanto pessoa e para avaliar a percepção de espaço apresentada pelo paciente.

O tempo é a duração entre um acontecimento e outro. É uma experiência única de cada indivíduo. É universal, por ser inerente aos processos de vida. É relacional, pois é individual e baseado na singularidade de cada pessoa e no seu ambiente perceptual. É unidirecional, movendo-se do passado ao futuro. É subjetivo, pois está baseado nas percepções pessoais sobre os sucessivos eventos de suas vidas. $O$ conhecimento da percepção de tempo que seus pacientes têm, ajudará as enfermeiras no atendimento às suas necessidades. $O$ tempo pode ser inerente às etapas da vida pessoal. Dimensões espaço-temporais do ambiente influenciam o sistema pessoal.

Todos esses conceitos relacionam seres humanos dinâmicos, que vivem em interação contínua com o ambiente e, com o aumento do número de indivíduos, aumenta também a complexidade das relações, o que ocorre no sistema interpessoa ${ }^{(4)}$ : Interações são os comportamentos observáveis em díades, tríades ou em grupos, em presença mútua. No processo interativo, dois indivíduos identificam metas e os meios para as alcançar mutuamente, sendo que uma das formas mais elevadas de interação é a fala. Quando uma pessoa interage com outra acontece uma ação, ao que vai decorrer uma reação, pois essa pessoa reage em presença da outra. 0 desenvolvimento dessa reação vai determinar se a interação continua ou não. Caso continue, será efetivada a transação. Nas situações de enfermagem é importante haver interação recíproca no estabelecimento de um sistema interpessoal positivo. A interação será influenciada pelo desempenho dos papéis individuais.

A Comunicação é considerada como o processo pelo qual uma informação é repassada de uma pessoa a outra, diretamente ou não. A função prioritária da linguagem em uma sociedade é facilitar a cooperação e interação entre indivíduos. Comunicação é um intercâmbio de pensamentos e opiniões entre indivíduos. Pode ser verbal, quando satisfaz desejos de reconhecimento, participação e auto-realização no contato direto entre as pessoas, ou não verbal, quando inclui gestos, expressões faciais, ações e posturas de ouvir e sentir ${ }^{(4)}$. 0 cuidado de enfermagem envolve conhecimento e habilidades de comunicação com uma variedade de indivíduos, buscando alcançar metas, que ocorrem na presença de transação.

Transações são comportamentos humanos dirigidos a metas. Acontecem em situações nas quais os seres humanos participam ativamente em eventos e esta participação ativa nos movimentos/ações para alcançar uma meta provoca mudança nos indivíduos. As transações não estão relacionadas ao movimento de "análise transacional". A interação humana conduz a transações, que reduzem 0 estresse em uma situação. A transação é sempre necessária por ocasião da formação de díades no sistema interpessoal.

Papel, como um constructo, é pertinente a cada um dos três sistemas em interação dinâmica neste modelo conceitual. No sistema interpessoal são identificadas relações interativas e modos de comunicação. $O$ conceito de papel exige que os indivíduos se comuniquem uns com os outros e interajam com o propósito de alcançar metas. O papel pode ser aprendido na família ou com outros grupos sociais. É situacional, pois depende da situação vivenciada e das pessoas envolvidas na interação. 0 enfermeiro é instruído no seu papel durante sua formação nas escolas de enfermagem e, mais tarde, ao participar de seu grupo profissional. 0 papel pode ser definido como o conjunto de comportamentos esperados daqueles que ocupam uma certa posição no sistema social, ou como o conjunto de procedimentos ou regras que definem as obrigações e direitos inerentes a uma posição organizacional. 0 conhecimento do papel é 
importante às enfermeiras para facilitar seu desempenho nos sistemas de cuidado à saúde. $O$ conflito de papéis em um sistema interpessoal, ou seja, a não adequação do eu ao papel a ser desempenhado, pode aumentar a tensão ou estresse no ambiente, interferindo de forma positiva ou não nas interações humanas.

Estresse é, em tese, um estado de dinamicidade no qual indivíduos interagem com o ambiente em busca de um equilíbrio que propicie o crescimento, desenvolvimento e desempenho efetivo de papéis. Envolve trocas de energia e informação entre as pessoas e 0 ambiente, possibilitando a regulagem e controle dos estressores. Mas deve ser controlado porque o estresse excessivo é prejudicial à saúde. A convivência das enfermeiras com a morte pode levá-las ao estresse. Algumas das características do estresse são: 1.universalidade, pois não está limitado a um tempo ou lugar; 2.dimensão espaço-temporal, porque a elevação excessiva do estresse acontece em momento $e$ situação determinados; 3.subjetividade, por variar de acordo com a forma como as pessoas respondem (reagem) aos eventos da vida. Pode-se dizer, então, que as enfermeiras estão numa posição de observadoras de padrões de respostas a estressores, pois, identificadas as causas da tensão, torna-se possível a intervenção.

Nas experiências vivenciadas pelas enfermeiras, uma base de conhecimento sobre sistemas sociais é essencial, pois provê a estrutura para a definição de relações sociais e estabelece regras de comportamento, modo e ação. O sistema social, é um sistema de limite organizado de papéis sociais, comportamentos e práticas desenvolvidas para manter valores e mecanismos de regulação dessas reações ${ }^{(4)}$. Os conceitos relevantes que a teórica implica nesse sistema são:

Organização, que é a forma pela qual as atividades contínuas são administradas para alcançar metas. Nela, os seres humanos despendem a maior parte de suas vidas, pois os indivíduos e grupos são suas unidades sociais. 0 entendimento da estrutura organizacional ajuda as enfermeiras na identificação e no enfrentamento dos conflitos entre a organização e seus papéis e funções profissionais. Está associada ao conhecimento de autoridade e poder.

Autoridade é o poder para tomar decisões que guiam as ações do self e de outros. É um processo ativo e recíproco de transação em que conhecimento, experiência, percepções e valores dos sujeitos influenciam a definição, confirmação e aceitação dos que se encontram em posições organizacionais, associados a esse poder. É essencial à realização de metas e às organizações, especialmente as formalmente constituídas. Mas é válido ressaltar que deve ser reconhecida por quem sofre sua influência, aceitando e obedecendo. Nos diversos sistemas de cuidado à saúde nos quais as enfermeiras trabalham, há a necessidade de entender a autoridade organizacional vigente, assim como a autoridade legítima da enfermeira. Sua apropriada utilização em uma organização promove satisfação ao trabalhador, eficiência, elevação moral e facilita o alcance de metas.

O poder é uma característica da autoridade, sendo 0 processo através do qual uma ou mais pessoas influenciam as demais numa determinada situação. 0 poder existe dentro das relações sociais e os que 0 exercem podem controlar grupos e organizações.

Status é como a posição de um indivíduo em um grupo é percebida por outros indivíduos. É o prestígio adquirido ao desempenhar um papel. Assim, o papel de enfermeira tem mais prestígio que o do auxiliar de enfermagem e é visto como de maior status.

Tomada de decisão é um conceito chave em qualquer organização. Decisões são julgamentos feitos que afetam o curso da ação a ser desenvolvida em situações específicas. Tomada de decisão tornou-se um conceito importantíssimo para as pessoas em todos os aspectos da vida. A própria ação ou resposta humana é decorrente de um processo de tomada de decisão. As decisões são situacionais e dirigidas a metas, compondo um processo contínuo, que envolve uma situação, estado ou problema. Constantemente, enfermeira e paciente tomam decisões sobre metas a serem alcançadas. Faz-se, então, necessária a informação compartilhada entre eles, respeitando os aspectos éticos. A vida humana é um contínuo processo de tomada de decisões, nas situações de vida e morte, sendo necessário conhecimento e habilidades para que sejam consideradas.

É possível afirmar, diante da descrição conceitual exposta, que as enfermeiras e os pacientes são seres humanos reativos que interagem em situações específicas para alcançar propósitos específicos. Imogene King não detém sua preocupação apenas sobre o paciente hospitalizado, mas também sobre indivíduos com doenças crônicas e sobre os que precisam de reabilitação. As enfermeiras cuidam de todos esses indivíduos, procurando capacita-los a utilizar sua habilidade potencial para funcionarem como seres humanos, desenvolvendo-se ao máximo no desempenho de seu papéis cotidianos $^{(4)}$.

Em um artigo mais recente ${ }^{(5)}$, a teórica afirma que a meta da utilização de seu modelo conceitual de sistemas é a saúde e que as funções desses sistemas são verificar ou observar nas relações recíprocas de indivíduos, como eles interagem e fazem transações com outras pessoas. A autora afirma que no seu modelo conceitual é possível identificar conceitos essenciais ao conhecimento da enfermagem enquanto disciplina. Permite também sua utilização como estrutura para desenvolvimento de um currículo para enfermagem e para implementar uma prática com base teórica que permita desenvolver um cuidado de qualidade aos indivíduos nos hospitais, ambulatórios e comunidades. 
$\mathrm{Na}$ Teoria de Alcance de Metas, propõe que a saúde/doença humana seja determinada pela interação dos três sistemas de seu modelo conceitual ${ }^{(4)}$, como observado na Figura 2.

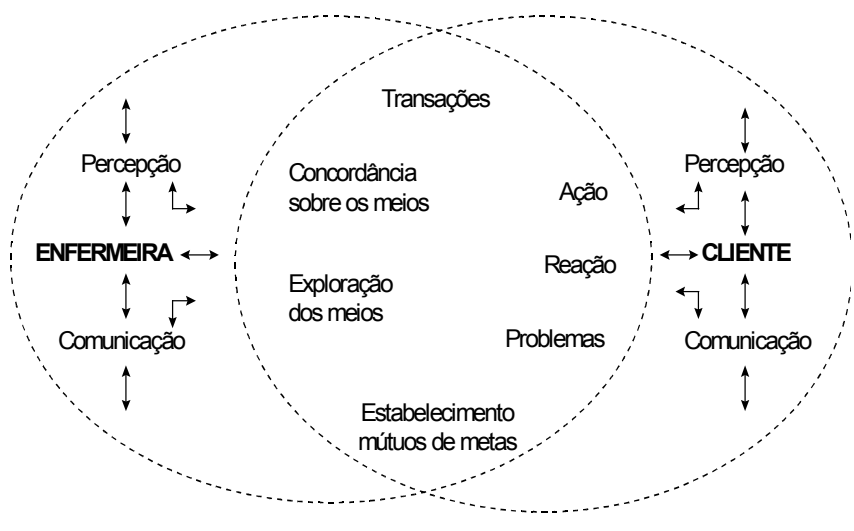

Figura 2 - A teoria do alcance de metas

0 processo de enfermagem de King compreende: 1.Interação inicial, que é um contato inicial que induz uma reação entre a enfermeira e o paciente. É extremamente influenciada pela percepção que um tem do outro e pela comunicação estabelecida entre eles; 2.Diagnóstico, que é a detecção das necessidades de cuidado dos seres humanos com vistas a alcançar a saúde, devendo ser confirmado com os pacientes; 3.Estabelecimento de metas comuns à díade enfermeira-paciente, com base nos diagnósticos detectados e em comum acordo das partes; 4. Exploração e viabilização de meios para alcançar as metas traçadas, devendo haver concordância entre ambas as partes sobre estes meios; 5 . Evolução, que é a avaliação contínua do alcance de metas, ressaltando fatores intervenientes nos três sistemas, para desenvolver a capacidade do paciente de enfrentar problemas, desenvolvendo-se bem nos papéis cotidianos $^{(4)}$.

Esse processo será permeado de ação e reação, uma vez que, os indivíduos são seres reagentes, e pautado no diagnóstico e na busca da resolução dos problemas, que somente será alcançada se ocorrer uma interação efetiva enfermeira-paciente ${ }^{(4)}$.

A meta dessa teoria é ajudar os indivíduos a manter um estado saudável e, assim, ajudá-los a desempenhar suas funções na sociedade. Os meios para alcançar uma meta comum variam em cada grupo profissional e de acordo com seus papéis e funções na sociedade. A meta global das enfermeiras é promover a saúde, prevenir a doença e se preocupar com o doente ${ }^{(4)}$.

Um registro sistemático do processo de implementação do plano de cuidado de enfermagem e dos resultados alcançados deve fazer parte do registro dos pacientes. As enfermeiras devem dar ênfase às metas a serem alcançadas na situação de enfermagem, aproximando-as da realidade e ajudando a verificar as percepções dos pacientes e a precisão dos dados levantados. Para isto, pode ser utilizada uma lista de problemas e também uma lista de metas mútuas fixadas.

Uma lista de problemas é um guia para avaliação contínua de sinais subjetivos e objetivos e de sintomas de uma perturbação ou interferência na habilidade do paciente para funcionar em seus papéis habituais. Serve como guia para identificar um diagnóstico de enfermagem e planejar o cuidado de enfermagem imediato ao paciente. A relação de problemas é utilizada pelas enfermeiras para guiar a implementação do plano de cuidado.

Listar ou relacionar metas serve para guiar a enfermeira a monitorar as perturbações ou interferências no paciente e estar alerta para qualquer nova informação relativa a este. As metas disponibilizam meios para a enfermeira e os pacientes interagirem, compartilharem informações, estabelecerem focos mútuos de atenção, explorarem meios e concordarem com eles para alcançar os objetivos. $O$ estabelecimento de prioridades permite a continuidade do cuidado e enfoca a participação dos pacientes nas decisões sobre seu cuidado. Seu benefício é a provisão de uma aproximação consistente e sistemática para ajudar os indivíduos a alcançar um estado saudável. Esta aproximação propicia o desenvolvimento do cuidado de enfermagem individualizado, facilitando a contínua colaboração do paciente na viabilização de meios para alcançar as metas traçadas ${ }^{(4)}$. 0 julgamento da evolução do paciente deve se dar pelo alcance das metas traçadas, permitindo a avaliação da evolução e a redefinição de metas, quando necessário.

A teoria de King regulamenta uma base teórica para 0 processo de enfermagem e fornece conhecimento básico de enfermagem como um processo de interações que conduzem a transações. Um registro meta-orientado facilita o uso desta teoria. Além disso, a teoria propicia uma aproximação sistemática para a enfermagem fazer diagnósticos das respostas do paciente. 0 diagnóstico de enfermagem identifica os problemas e as necessidades dos pacientes, que podem ser registrados em um formulário. A partir desse levantamento é desenvolvido um instrumento, que identifica metas a curto, médio e longo prazo. Os resultados da evolução são julgados em termos do alcance dessas, sendo as alcançadas mensuradas pela efetividade do cuidado de enfermagem, servindo para avaliação de sua qualidade. Seu registro pode fornecer uma estrutura para colaboração e cooperação junto a outros profissionais, possibilitando que o cuidado possa ser avaliado.

A operacionalização de seu processo de enfermagem se dá da seguinte maneira: existência de uma interação inicial, em cujo curso devem ser compartilhadas informações, realizados diagnósticos, fixadas metas mútuas e viabilizados meios de alcance dessas; implementação de um plano de enfermagem, que passará por avaliação contínua da evolução pelas interações enfermeirapaciente, pelas observações da enfermeira e pelas medidas de 
variáveis biológicas, psicológicas, dentre outras. Durante todas as etapas, deve se solicitar aos pacientes que participem das decisões.

$\mathrm{Na}$ interação inicial poderá ser utilizado um roteiro para anamnese. A partir da aplicação desse instrumento serão detectadas alterações, problemas e preocupações relacionados ao cotidiano dos pacientes, devendo ser considerados apenas os diagnósticos que forem confirmados por estes. Poderão ser definidas metas comuns entre a enfermeira e o paciente a curto, médio e longo prazos, e estabelecidas intervenções de enfermagem. Os pacientes devem ser atendidos em consulta de enfermagem periódica para avaliação de sua evolução, quando pode ocorrer ou não uma redefinição de metas. Assim, deve-se fazer, de modo centrado numa ação conjunta com o paciente: 1. registro de enfermagem; 2 . determinação dos problemas; 3. definição das metas; 4 . viabilização de meios para seu alcance; 5 . implementação, em comum acordo com o paciente, das ações definidas; 6 . julgamento da evolução do paciente em termos do alcance das metas e 7 . redefinição do plano, quando necessário.

\section{REFLETINDO SOBRE O CUIDAR A PARTIR DA ESTRUTURA DE KING}

Atualmente, a utilização de um modelo de sistemas parece ser ideal, pelas mudanças e complexidade das organizações de saúde, sendo, provavelmente, o modo mais adequado de estudar seres humanos em interação com o ambiente por desenvolver uma estrutura conceitual de variáveis mutuamente dependentes e conceitos relacionados. Vimos que o modelo conceitual de King é a armação estrutural básica sobre a qual se desenvolve sua Teoria de Alcance de Metas e que esse é composto dos sistemas pessoal, interpessoal e social, preconizando o estabelecimento de metas comuns na díade enfermeira-paciente e a determinação de meios que viabilizem seu alcance.

Algumas críticas têm sido feitas ao modelo e à teoria de King, destacando-se a limitação de sua aplicação junto a pacientes sem condições de interagir competentemente com a enfermeira, questionando até que ponto são responsáveis pelas decisões relativas ao tratamento, enfatizadas nesta teoria ${ }^{(6)}$.

No entanto, outros autores reforçam a viabilização de aplicação desta teoria na prática, pelo fornecimento de uma estrutura na qual é apoiado o pensamento crítico da enfermeira ${ }^{(7)}$. É afirmado também que a estrutura conceitual e a teoria elaboradas por King não se limitam à cultura norte-americana, podendo ser aplicadas à prática profissional de enfermagem de qualquer cultura e sociedade humana ${ }^{(8)}$. E que os conceitos sistematizados da estrutura conceitual de King proporcionam uma forma atual e válida de compreender os seres humanos ${ }^{(9)}$, uma vez que o processo de enfermagem de King ${ }^{(4)}$ é permeado de ação e reação, pela própria característica interacionista dessa teoria ${ }^{(1)}$. Esta teoria pode ser utilizada ainda para guiar o processo ensino-aprendizagem em enfermagem ${ }^{(10)}$.

Os conceitos desse modelo foram utilizados para compreender o comportamento de não adesão de pacientes com hipertensão arterial (HA) por ocasião de nossa dissertação de mestrado ${ }^{(11)}$ e a utilização da teoria foi testada em um estudo de caso $^{(12)}$. Entendemos que a necessidade de adesão ao tratamento da HA requer uma interação efetiva, que pode se dar pelo estabelecimento de metas mútuas fixadas com cada paciente, que, em decorrência da doença, sofre alterações nos seus sistemas.

Acreditamos que tal modelo conceitual, assim como essa teoria, tenham aplicação junto a pacientes com outras doenças crônicas, que apresentam uma evolução lenta, o que pode desestimulá-los com relação ao tratamento. Trabalhar com metas pode encorajá-los a prosseguir e, com o apoio da família, esse encorajamento pode atingir o controle da doença, prevenindo complicações e dando-lhes condições de uma vida melhor. A interação entre os sistemas pessoal, interpessoal e social será determinante nesse processo, pois o paciente passa a ser visto como um sistema pessoal extremamente influenciado pelas pessoas com quem convive e pelo meio no qual vive. Assim, a Teoria de Alcance de Metas de King é, não somente aplicável ao trato de tais pacientes, mas desejável, pois viabiliza uma forma de interação paciente-enfermeiro necessária à eficácia terapêutica, que sabemos, requer um cuidado mais pessoal.

A tendência observada atualmente e já evidenciada nas abordagens das pesquisas mais recentes é de uma visão mais humanista na enfermagem, embora essa não deva prevalecer nas ações de enfermagem, rejeitando os aspectos científico e técnico como esses eram anteriormente privilegiados com exclusividade ${ }^{(13)}$. Novas proposições se formam para repensar o cuidado, colocandoo ao lado do paciente, e não da doença, tentando escapar da exclusividade biológica, pondo em ação novas lógicas que repousam na interdependência e não na hierarquização multiprofissional ${ }^{(14)}$.

Historicamente, o cuidado tem sido repassado para os profissionais de saúde com foco na cura do corpo doente, percebido de forma fragmentada, como objeto ou máquina. Isto é muito negativo e pode gerar como resultado um processo de desumanização e embrutecimento das relações, que repercute no atendimento aos pacientes ${ }^{(15)}$. Ir de encontro a este embrutecimento nas relações com os pacientes deve ser meta dos profissionais de saúde, especialmente no cuidado de doentes crônicos, que requerem um controle terapêutico sistemático e permanente. É importante oferecer um atendimento humanizado, no qual a família esteja inserida como agente de cuidado. A família já não pode ser vista apenas como a cumpridora das determinações dos profissionais de saúde ${ }^{(16)}$. Ela precisa ser ouvida, ter sua opinião considerada e sua participação incentivada em todo o processo profissional de cuidar/curar. 


\section{REFERÊNCIAS BIBLIOGRÁFICAS}

1. Barnum BJS. Nursing Theory: Analysis, Application, Evaluation. 5.ed. New York: Lippincott; 1998.

2. George JB, organizador. Teorias de enfermagem: fundamentos para a prática profissional. Porto Alegre: Artes Médicas; 2000.

3. Garcia TR, Pagliuca LMF. A construção do conhecimento em enfermagem: coletânea de trabalhos. Fortaleza: RENE; 1998.

4. King IM. A theory for nursing: systems, concepts, process. New York: Wiley Medical Publications; 1981.

5. King IM. King's Theory of Goal Attainment in Practice. Nursing Science Quarterly: theory, research, and practice 1997; 10(4):180-5.

6. Dióz M, Oliveira AGB de. Teoria de alcance dos objetivos de Imogene King: análise crítica. Col de Enfermagem. FEN/UFMT 1999 jan.jjun; 1(1):215-25.

7. Frey MA, Norris D. King's systems framework and theory in nursing practice. In: Alligoood MR, Marriner-Tomeyy A. Nursing theory: utilization \& application. St. Louis-Missouri: Mosby-Year Book; 1997. p. $71-88$.

8. Garcia TR. Cuidando de adolescentes grávidas solteiras. [tese]. Ribeirão Preto (SP): Escola de Enfermagem de Ribeirão Preto/USP; 1996.

9. Resende MMC. Fatores que dificultam o controle da hipertensão arterial à luz da estrutura conceitual da Teoria de King. [dissertação]. Belo Horizonte (MG): Escola de Enfermagem/UFMG; 1998.
10. Dal Bello ITR. Processo ensino-aprendizagem com alunos do curso de graduação em enfermagem com base na teoria do alcance dos objetivos de Imogene King. [dissertação]. Concórdia (SC): Departamento de Enfermagem/Universidade Federal de Santa Catarina; 2000.

11. Moreira TMM. Descrevendo a não adesão ao tratamento a partir de uma compreensão de sistemas. [dissertação]. Fortaleza (CE): Departamento de Enfermagem da Faculdade de Farmácia, Odontologia e Enfermagem/Universidade Federal do Ceará; 1999.

12. Moreira TMM, Araújo TL. A teoria de alcance de metas de Imogene King no atendimento a um cliente com hipertensão não aderente ao tratamento. In: $5^{\circ}$ Encontro de iniciação à pesquisa da UNIFOR; 1999. Anais. Fortaleza (CE): EDUFC; 1999. p. 217.

13. Waldow VR. Cuidado humano: o resgate necessário. Porto Alegre: Sagra Luzzatto; 1998.

14. Lopes MJM. A singularidade de um saber-fazer técnico e relacional. In: 50 Congresso Brasileiro de Enfermagem: Cuidar: ação terapêutica da enfermagem; 1998. Anais. Salvador (BA): ABEn-BA; 1999. p. 66-73.

15. Silva AL. Cuidado transdimensional: um paradigma emergente. Pelotas: Ed. Universitária/UFPEL; Florianópolis: Programa de PósGraduação em Enfermagem/ UFSC; 1997. Série Teses de Enfermagem, n. 6.

16. Elsen I. Desafios da enfermagem no cuidado de famílias. In: Elsen I. Marcos para a prática de enfermagem com famílias. Florianópolis: EDUFSC; 1994. 